\title{
Whole genome sequencing and identification of Bacillus endophyticus and $B$. anthracis isolated from anthrax outbreaks in South Africa
}

Kgaugelo Edward Lekota ${ }^{1,2,3,4}$ (D), Oliver Keoagile Ignatius Bezuidt ${ }^{1}$, Joseph Mafofo ${ }^{1}$, Jasper Rees ${ }^{1}$, Farai Catherine Muchadeyi ${ }^{1}$, Evelyn Madoroba ${ }^{3,4,5}$ and Henriette van Heerden ${ }^{2^{*}}$

\begin{abstract}
Background: Bacillus endophyticus is a soil plant-endophytic bacterium, while B. anthracis is the causative agent of anthrax. The virulence factors of $B$. anthracis are the plasmid encoded tripartite toxins ( $p X O 1)$ and poly- $\gamma$-glutamic acid (PGA) capsule (pXO2). B. endophyticus isolated alongside $B$. anthracis from animals that died of anthrax in Northern Cape Province (NCP), South Africa, harbored polyglutamate genes. The study compared the characteristics of $B$. anthracis and B. endophyticus with other Bacillus species with a focus on the presence of the PGA capsule or/ and unbound PGA. The morphology and whole genome sequence analysis of $B$. endophyticus strains and $B$. anthracis were compared.

Results: In conventional microbiology, B. endophyticus showed gram-positive round-shaped rods in single/short chains, which were endospore-forming, non-motile, non-haemolytic with white and dry colonies, and $\gamma$-phage resistant. B. anthracis was differentiated from B. endophyticus based on the latter's box-shaped rods in pairs/long chains, white-grey and slimy colonies, encapsulated and $\gamma$-phage susceptible. The study identified a PGA polyglutamate synthase operon that consisted of pgsBCA, $\gamma$-glutamyltranspeptidase (ggt) and pgsE in B. endophyticus genomes.

Conclusions: $\mathrm{PGA}$ regions of $B$. anthracis contain capBCADE genes located in the $\mathrm{pXO} 2$ required for capsulation formation, while $B$. endophyticus contain the pgsBCAE genes in the chromosome. Whole genome and microbiology analysis identified B. endophyticus, as a non-capsuled endospore-forming bacterium that consists of PGA required for biosynthesis. $B$. endophyticus strains do not synthesize surface associated PGA, therefore capsule visualization of B. anthracis is a key diagnostic characteristic. The study highlights the significance of using whole genome shotgun sequencing to identify virulence and other important genes that might be present amongst unknown samples from natural outbreaks. None of the $B$. anthracis related plasmids or virulence genes were found in the $B$. endophyticus genomes.
\end{abstract}

Keywords: Bacillus endophyticus, Bacillus anthracis, Poly-y-glutamic acid (PGA), Whole genome sequencing (WGS)

\footnotetext{
* Correspondence: Henriette.VanHeerden@up.ac.za

${ }^{2}$ Department of Veterinary Tropical Diseases, University of Pretoria, Private

bag X4, Onderstepoort 0110, South Africa

Full list of author information is available at the end of the article
}

(c) The Author(s). 2018 Open Access This article is distributed under the terms of the Creative Commons Attribution 4.0 International License (http://creativecommons.org/licenses/by/4.0/), which permits unrestricted use, distribution, and reproduction in any medium, provided you give appropriate credit to the original author(s) and the source, provide a link to the Creative Commons license, and indicate if changes were made. The Creative Commons Public Domain Dedication waiver (http://creativecommons.org/publicdomain/zero/1.0/) applies to the data made available in this article, unless otherwise stated. 


\section{Background}

Bacillus endophyticus is regarded as a plant-endophytic bacterium that is found in the inner tissues of plants, specifically cotton [1]. It is present either as gram-positive single rod-shaped cells or as chains that can be short or long, non-haemolytic and non-motile. Biochemical characteristics that differentiate $B$. endophyticus from other Bacillus species include the inability to reduce nitrate $\left(\mathrm{NO}^{3-}\right)$ to nitrite, casein and starch, as well as, ampicillin and $\mathrm{NaCl}$ resistance [1].

B. anthracis is the causative agent of anthrax, and primarily affects herbivorous animals, although all mammals can also be affected. The vegetative cells of B. anthracis appear 'box-shaped' either in pairs or chains. It is phenotypically characterized as gram-positive aerobic rods $(3-5 \mu \mathrm{m} \times 1 \mu \mathrm{m})$, that are non-haemolytic, non-motile, penicillin and $\gamma$-phage resistant [2]. However, it is distinguishable from its close relatives by its ability to synthesize virulence factors encoded on plasmids, pXO1 and $\mathrm{pXO} 2$. The pXO1 (182 kb) contains genes encoding for the tripartite anthrax toxins (protective antigen, lethal factor and edema factor) and pXO2 (96 kb) encodes a five-gene operon capBCADE (capsule biosynthesis genes), which synthesizes a poly- $\gamma$-glutamic acid (PGA) capsule $[3,4]$. Capsule biosynthesis genes are transcribed as a single operon predicted to encode proteins for the biosynthesis, transport and attachment of D-glutamatic acid residue on the bacterial surface [5]. The anthrax capsule activators $(a c p \mathrm{~A}$ and $a c p \mathrm{~B})$ located on $\mathrm{pXO} 2$ are controlled by anthrax toxin activator (atxA) located on pXO1 [5]. The PGA capsule enables host immune system evasion by protecting the vegetative cells from phagocytosis by macrophages [5]. The vegetative cells of $B$. anthracis have also been shown to secrete the PGA capsules under anaerobic conditions and in the presence of bicarbonate $[3,5]$.

Many pathogenic bacteria require a cell-associated capsule for virulence [6]. Capsule composition of bacteria can be in a form of polypeptide (poly-glutamate) or polysaccharide. Poly- $\gamma$-glutamic acid (PGA) is a poly-anionic polymer that may be composed of only $\mathrm{D}$, only $\mathrm{L}$ or both glutamate enantiomers [4, 7]. Most strains producing PGA are members of the gram-positive Bacillus group. The function of PGA depends on whether it is bound to peptidoglycan or unbound/released. In the bound state it forms the capsule, whereas in the secreted/unbound state it is released into the environment $[4,8]$. The uncommon bound PGA capsule only includes $B$. anthracis and Staphylococcus epidermidis that synthesize the anchored (surface-associated) PGA, which enables them to act as a virulence factor [4]. The B. anthracis PGA synthesis genes are encoded on the pXO2 consisting of $\operatorname{capB}, \operatorname{cap} \mathrm{C}, \operatorname{capA}$ and $\operatorname{cap} \mathrm{E}$, while cap $\mathrm{D}$ acts as the peptidoglycan binding/ anchoring site $[4,7,9]$. The corresponding polyglutamate biosynthesis pathway orthologs in B. subtilis includes $p g s \mathrm{~B}, p g s \mathrm{C}$ and pgsAA [10] and pgsS has been suggested to induce the release of PGA $[4,7]$. The capBCADE genes of $B$. anthracis encoded on pXO2 have functional orthologs encoded on the chromosomes of B. subtillis/licheniformis and other Bacillus species [4, 11]. Few species such as $B$. anthracis and $S$. epidermidis have been reported to produce the PGA capsule [4]. The unbound PGA have been reported to Bacillus species such as B. cereus strains ATCC 10987, 14,579 and B. thuringiensis 97-27, AI Hakam [4]. B. cereus biovar anthracis strains isolated from great apes that died of anthrax symptoms in west and central Africa were shown to harbor the B. cereus chromosome and pXO2-like plasmid [12] that contained the PGA capsule genes identical to those of $B$. anthracis.

Gene sequences that encode for the formation of PGA and capsules on the pathogenic and non-pathogeneic species need to be compared and distinguished from their close relatives [11]. This is essential especially when some of the virulence gene sequences and morphological characteristics are used for identification and diagnosis of anthrax. In this study, B. endophyticus strains were isolated alongside $B$. anthracis strains from animals that died of anthrax in Northern Cape Province (NCP), South Africa in an outbreak that occurred in 2009. B. endophyticus is regarded a plant-endophyte and it is uncommon to be isolated from blood or animals. The B. endophyticus strains that were isolated alongside $B$. anthracis strains had some of the similar morphological, biochemical and some genetic characteristics compared to the anthrax causing bacteria. In our previous study, conventional PCR detected PGA gene regions in both $B$. anthracis and B. endophyticus isolates and attempts were made to distinguish and identify these strains using routine and non-routine diagnostic methods [13]. The $B$. endophyticus strains were identified using non-routine diagnostic Omnilog (Biolog) and 16S rRNA sequencing methods and differentiated based on routine diagnostic microbiological tests and real time PCR. Therefore, in order to enhance and contribute to the unequivocal diagnosis of $B$. anthracis, the goal of this study was to perform comparative analysis of the $B$. endophyticus and B. anthracis strains from the afore-mentioned outbreak as well as contribute towards the scant genome information of $B$. endophyticus. Thus the virulence genes of $B$. anthracis occurring on the plasmids were investigated, as well as the capsule and phenotypic characteristic of related Bacillus species were summarized using results from this study and published literature to enhance and contribute towards anthrax diagnosis.

\section{Results}

\section{Phenotypic characterization}

B. endophyticus strains reported in the study were isolated from the environment and/or animals that died of 
B. anthracis during the 2009 anthrax outbreak in Northern Cape Province (NCP) (Table 1). On sheep blood tryptose agar (SBTA) at $5 \% \mathrm{CO}_{2}$, colonies of B. anthracis appeared whitish-grey, smooth, dry and shiny (medusa head), while $B$. endophyticus colonies were circular white, slimy or rough (Additional file 1: Figure S1 (2)). The B. endophyticus colonies on nutrient agar supplemented with $0.8 \%$ sodium bicarbonate at $5 \% \mathrm{CO}_{2}$ were smaller and circular, non-mucoid and wet (Additional file 1: Figure S1A), whereas B. anthracis colonies appeared circular, mucoid and shiny (Additional file 1: Figure S1B). Colony morphology of the B. endophyticus strains was observed after $24 \mathrm{~h}$ in culture compared to $B$. anthracis, which was observed earlier (12-24 h) on sodium bicarbonate supplemented nutrient agar.

Gram-positive B. anthracis cells occurred in box-shaped rods in pairs and/or long chain rods (Fig. 1a) that are encapsulated $\left(\mathrm{cap}^{+}\right)$after incubation at $5 \% \mathrm{CO}_{2}$ in blood (Fig. 1b), while the gram-positive B. endophyticus appeared as round-edged rods either as single and/ or short chains (Fig. 1c, Table 2). No capsules were observed in $B$. endophyticus strains after incubating at $5 \% \mathrm{CO}_{2}$ (Fig. 1d). B. anthracis 3631_1C [14] and B. anthracis Sterne strains were non-capsulated $\left(\mathrm{cap}^{-}\right)$since they lack pXO2 while $B$. anthracis 20SD was capsulated (Fig. 1e). The terminal ellipsoidal spores were also observed in B. endophyticus 3631_9D strain using the copper sulphate stain after $24 \mathrm{~h}$ incubation on nutrient agar containing $0.8 \%$ sodium bicarbonate (Fig. 1 and Additional file 2: Figure S2A-D).

The comparison of phenotypic properties of $B$. endophyticus, B. anthracis, B. cereus B. megaterium and $B$. smithii strains is shown in Table 2. B. anthracis and $B$. cereus were compared in Table 2 as they belong to $B$. cereus sensu lato group, while $B$. megaterium is closely related to $B$. endophyticus based on whole genome sequence and some of the microbiological features are similar to $B$. anthracis. B. smithii is a closely related species of $B$. endophyticus based on 16S rRNA sequence gene. However, Table 2 shows that $B$. cereus and $B$. smithii are both motile and can easily be excluded from B. anthracis. B endophyticus is a gram-positive, non-capsulated, non-motile, round-edged rod that is endospore-forming, non-hemolytic, penicillin sensitive but $\gamma$-phage resistant bacterium. $B$. anthracis is a gram-positive capsulated, non-motile, box-shaped rod that is endospore-forming, non-hemolytic, penicillin and $\gamma$-phage sensitive (Table 2). In this study biochemical characterization showed some common results between B. anthracis and B. endophyticus including the positive reaction for catalase and oxidase and negative reaction for indole (Table 2). Biochemical properties of B. endophyticus that differentiated it from other Bacillus species included inability to reduce nitrate to nitrite, hydrolyze casein, gelatin, and starch, as well as resistance to $\mathrm{NaCl}$. The absence of lecithinase and Voges Proskaeur (VP) can be used to distinguish B. endophyticus from B. anthracis (Table 2).

\section{S rRNA gene phylogenetic analysis}

The 16S rRNA gene sequences of B. endophyticus strains 3631_9D, 3617_2C, 3631_10C and 3618_1C strains were used to mine for other $16 \mathrm{~S}$ rRNA gene sequences through BLAST homology searches. The sequenced $B$. endophyticus strains 3631_9D, 3617_2C and 3631_10C showed a $100 \%$ similarity with the $16 \mathrm{~S}$ rRNA gene sequences of B. endophyticus strains (A6, S160(2), 2DT and uncultured bacterium 12TR2ACLN347) (Additional file 3: Figure S3). Strain 3618_1C grouped with majority of the uncultured bacterium (12TRACLN435 and 12TRACLN431) obtained from NCBI. The B. cereus sensu lato group grouped separately from the B. endophyticus based on 16S rRNA gene region (Additional file 3: Figure S3).

\section{Average nucleotide identities, pan-genome analyses functional classification of orthologous genes}

South African B. endophyticus sequences (3617_2C, 3618_1C, 3631_9D, 3631_10C) had a total of approximately 5.1 to 45.3 million reads with an average length

Table 1 Bacillus endophyticus and B. anthracis strains isolated from animal anthrax cases in Northern Cape province (NCP) in South Africa

\begin{tabular}{|c|c|c|c|c|c|}
\hline Strain number & Animal source ${ }^{c}$ & Specimen & Isolation date & $\begin{array}{l}\text { Provinces and location } \\
\text { (farm or town) in South Africa }\end{array}$ & Bacillus species \\
\hline $3617 \_2 C^{a}$ & Tragelaphus strepsiceros (Kudu 1) & Ear blood & May-09 & NCP (Klipfontein) & B. endophyticus \\
\hline $3618 \_1 C^{a}$ & Tragelaphus strepsiceros (Kudu 2) & Ear blood & May-09 & NCP (Kimberly) & B. endophyticus \\
\hline 3631_9D ${ }^{a}$ & Ovis aries (Sheep 1) & Ear blood & May-09 & NCP (Kimberly) & B. endophyticus \\
\hline $3631{ }_{10} C^{a}$ & Ovis aries (Sheep 2) & Ear blood & May-09 & NCP (Kimberly) & B. endophyticus \\
\hline 3618_2D & Tragelaphus strepsiceros (Kudu 2) & Soil & May-09 & NCP (Klipfontein) & B. anthracis \\
\hline $3631 \_1 C^{b}$ & Tragelaphus strepsiceros (Kudu 3) & Ear blood & May-09 & NCP (Klipfontein) & B. anthracis \\
\hline 3617_1C & Tragelaphus strepsiceros (Kudu 1) & Ear blood & May-09 & NCP (Klipfontein) & B. anthracis \\
\hline
\end{tabular}

${ }^{\mathrm{a}}$ The strains that were subjected for sequencing

${ }^{b}$ Draft genome from previous study [14]

${ }^{c}$ Kudu 1, kudu 2, sheep 1 etc. refer to different animal carcasses with B. endophyticus (3617_2C) and B. anthracis (3617_1C) isolated from the same kudu carcass (kudu 1) 


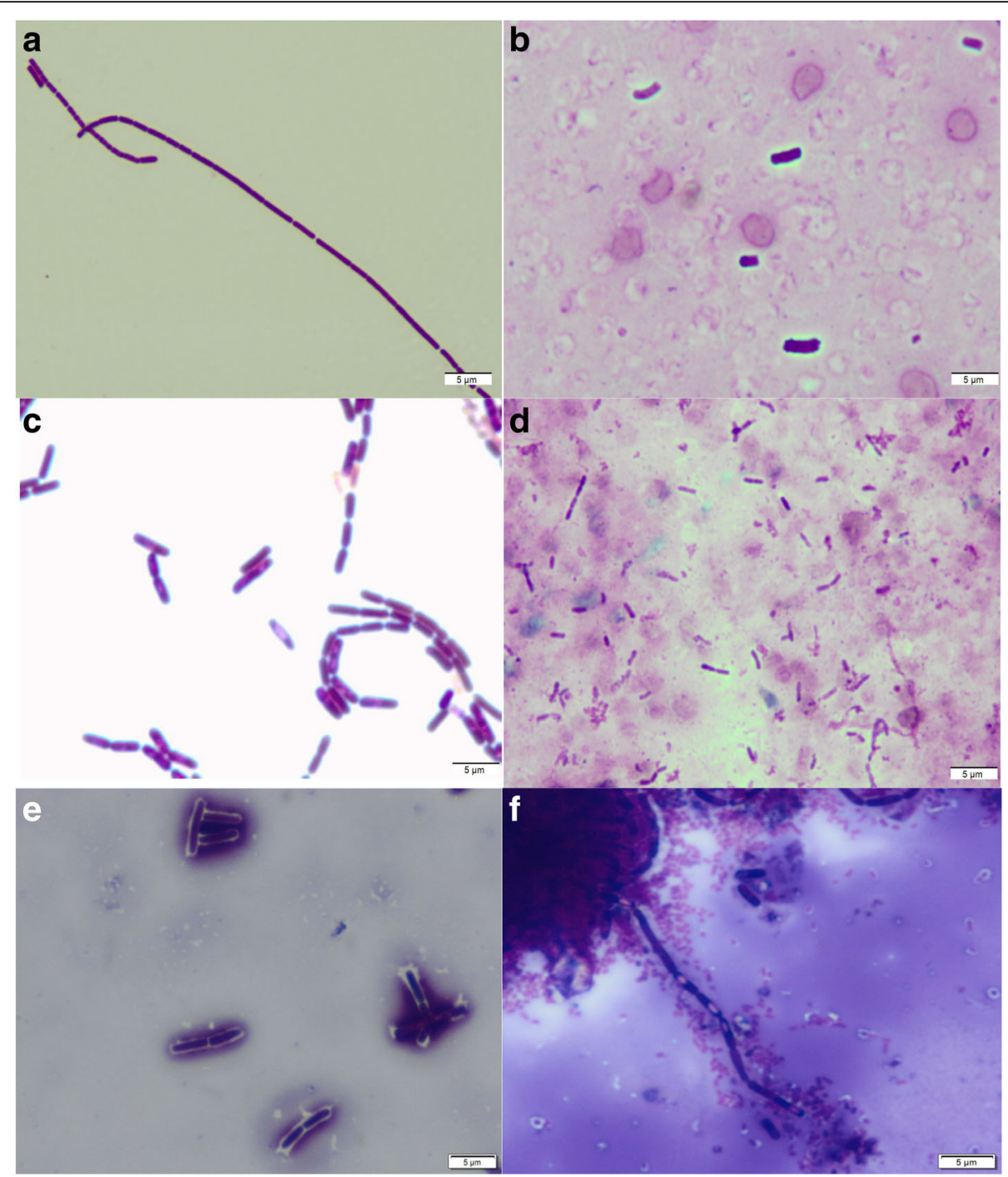

Fig. 1 Phenotypic electron microscopic examination of the morphology of Bacillus anthracis and B. endophyticus strains. (a) Gram-positive vegetative cells of B. anthracis Sterne long, bacilli chains, (b) B. anthracis 3618_2D capsulated in blood serum, (c) Gram-positive vegetative cells of B. endophyticus short, bacilli chains, and (d) B. endophyticus 3631_9D non-capsulated in blood, (e) capsulated B. anthracis 3618_2D and (f) B. endophyticus 3631_9D non-capsulated with spores after incubation on nutrient agar containing $0.8 \%$ sodium bicarbonate in the presence of $5 \% \mathrm{CO}_{2}$

of 94 nucleotides after trimming. Sequenced reads were de novo assembled (Table 3) and annotated using PGAAP for further classification of the $B$. endophyticus strains. The heat map (Fig. 4) indicated the average nucleotide identities of the B. endophyticus CDSs of South African sequenced strains and available whole genome sequences (2102, Hbe603, A6, S160(2), 2DT, KCC 13922, DSM13796 and uncultured bacterium 12TR2ACLN347). The sequenced B. endophyticus strains in this study as well as $B$. endophyticus DSM 13976 and KCTC 13922 had the same profile (with an ANI score of >98\%); B. endophyticus 3617 2C is highly related with this two genomes forming their own sub-clade, but clustered separate from B. endophyticus 2102 and Hbe603 strains (Fig. 2). B. endophyticus
$3618 \_1 C$ grouped separately amongst the sequenced $B$. endophyticus strains.

The pan-genome homology analysis of 4 South African B. endophyticus and Hbe603 strains identified 7154 clusters of protein coding genes with 3711, 3954, 997 and 2203 clusters represented the core, softcore, shell and cloud genomes, respectively (Fig. 3). In this study the $B$. endophyticus has more genes assigned to the core than the accessory genes (shell and cloud clusters), but the latter might increase when more genomes are sequenced and become available (Fig. 3). In the COG category assignments, the core and the accessory genomes have a slightly different number of genes assigned to the defense mechanisms category (Fig. 3 
Table 2 Comparison of phenotypic and biochemical characteristics of Bacillus endophyticus, B. anthracis, B. cereus, B. megaterium and B. smithii

\begin{tabular}{|c|c|c|c|c|c|}
\hline Microbiological characteristics & B. endophyticus & B. anthracis & B. cereus & B. megaterium & B. smithii \\
\hline Gram reaction & + & + & + & + & + \\
\hline Rods $(\mu \mathrm{m})$ & $2.5-3.5 \times 0.5-1.5$ & $3-6 \times 1-1.25$ & $3-5 \times 1-1.2$ & $2-5 \times 1.2-1.5$ & $5-6 \times 0.8-1.0$ \\
\hline Spores & Ellipsoidal/cylindrical & Ellipsoidal/cylindrical & Ellipsoidal/cylindrical & Ellipsoidal/Spherical & Ellipsoidal/cylindical \\
\hline Haemolysis & $\mathrm{NH}$ & $\mathrm{NH}$ & $\mathrm{H}$ & $\mathrm{H}^{\%} \%$ & ND \\
\hline Motility & Non-motile & Non-motile & Motile & Non-motile & motile \\
\hline Capsule & - & + & - & + & - \\
\hline Penicillin & $S$ & $S$ & $\mathrm{R}$ & ND & ND \\
\hline Gelatin hydrolysis & - & $+{ }^{*}$ & + & + & - \\
\hline Nitrate reduction & - & + & + & $(-)$ & - \\
\hline Starch hydrolysis & - & + & + & + & W+ \\
\hline Voges Proskaeur (VP) & - & + & + & - & - \\
\hline$\gamma$-phage & $\mathrm{R}$ & $s^{\$}$ & R & $S^{@}$ & ND \\
\hline Casein & - & + & + & + & - \\
\hline Egg yolk/Lecithinase & - & + & + & - & - \\
\hline Urea hydrolysis & - & - & - & $-{ }_{-1} \%$ & - \\
\hline Citrate & W+ & - & + & + & $(-)$ \\
\hline $\mathrm{NaCl}$ & $2-10 \%$ & $5 \%$ & $2-7 \%$ & $7 \%$ & $2-3 \%$ \\
\hline Catalase & + & + & + & + & + \\
\hline Indole & - & - & - & - & - \\
\hline Oxidase & + & + & - & $(+)$ & + \\
\hline
\end{tabular}

Abbreviations: +, positive, $\mathrm{W}+$, weakly positive, - , negative, (), variable, $\mathrm{S}$, susceptible, $\mathrm{R}$, resistance, $\mathrm{ND}$, not determined, $\mathrm{NH}$, non-haemolytic, ${ }^{*}$, inverted fir tree, ${ }^{\$}$, B. anthracis strains resistance to $\gamma$-phage have been reported [22], @ resistant $\gamma$-phage B. megaterium strain has been reported at Kansas State University [44] . All data of $B$. endophyticus were obtained in the study and supplemented with information of Reva et al. [1]. B. anthracis and $B$. cereus information was compiled from WHO [42]; \%\% Beesley et al. [27] reported B. megaterium non-haemolytic and urea hydrolysis strains

Table 3 Genome comparison features of the Bacillus endophyticus strains used in the study

\begin{tabular}{|c|c|c|c|c|c|}
\hline & $\begin{array}{l}\text { B. endophyticus } \\
\text { 3631_9D }\end{array}$ & $\begin{array}{l}\text { B. endophyticus } \\
3618 \_1 C\end{array}$ & $\begin{array}{l}\text { B. endophyticus } \\
3631 \text { 10C }\end{array}$ & $\begin{array}{l}\text { B. endophyticus } \\
3617 \_2 C\end{array}$ & $\begin{array}{l}\text { B. endophyticus } \\
\text { Hbe603 }^{\mathrm{a}}\end{array}$ \\
\hline Genome size (bp) & $5,311,808$ & $5,379,838$ & $5,243,706$ & $5,319,031$ & $5,313,985$ \\
\hline GC content (\%) & 36 & 36 & 36 & 36 & 36 \\
\hline Total contigs & 57 & 68 & 60 & 99 & 9 \\
\hline N50 & 304,287 & 152,600 & 277,642 & 99,724 & $4,865,574$ \\
\hline Maximum contig & 820,841 & 657,546 & 874,718 & 224,909 & NA \\
\hline Minimum contig & 307 & 141 & 1164 & 227 & NA \\
\hline Coding sequences $(C D S)^{b}$ & 5310 & 5470 & 5358 & 5408 & 5455 \\
\hline RNAs & 68 & 54 & 50 & 47 & 114 \\
\hline Prophage regions & 1 & 7 & 2 & 2 & 4 \\
\hline Chromosome (size) & $5,056,260$ & $5,011,594$ & $5,305,974$ & $5,231,075$ & $4,865,574$ \\
\hline Number of plasmids ${ }^{c}$ & 7 & 4 & 6 & 6 & 8 \\
\hline
\end{tabular}

${ }^{a}$ Bacillus endophyticus Hbe603 was used as a reference strain in this study

${ }^{\mathrm{b}}$ Coding sequences predicted using RAST

'BLASTn with an e-value of $1 \mathrm{e}-10$ and $>90 \%$ identity was used against the $B$. endophyticus Hbe603 plasmids

$N A$ not available 


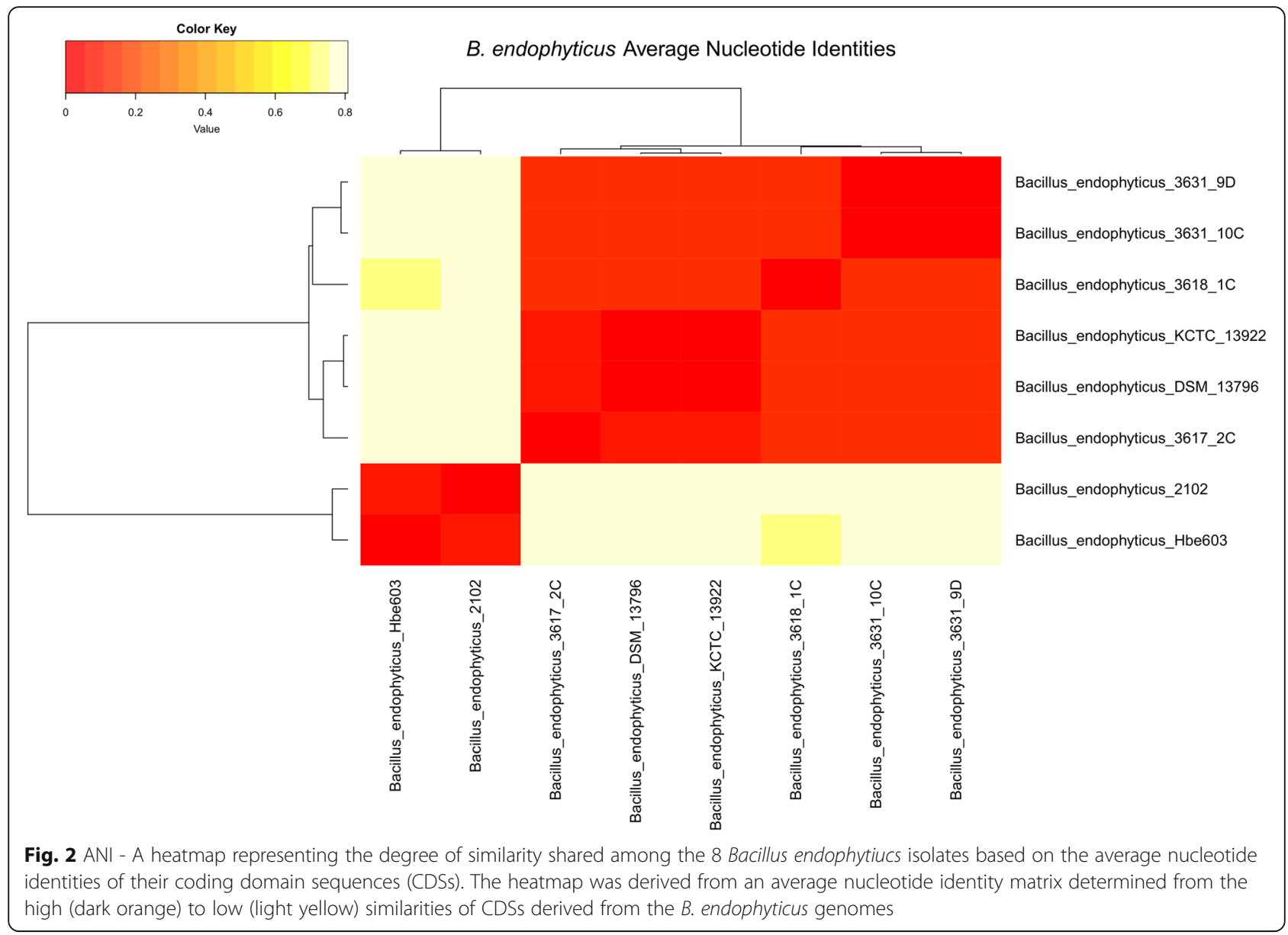

category V) as in most cases this category is mainly abundant in the accessory genome [15]. The core cluster dominates all the other categories, including categories for function unknown (S) and general prediction only $(R)$ in Fig. 3.

\section{Genomic features of $B$. endophyticus strains}

Comparative genomics of the draft sequenced $B$. endophyticus strains in the study and complete genome $B$. endophyticus Hbe603 showed almost equivalent genome sizes with the complete genome of $B$. endophyticus Hbe603 (Table 3). The GC content of the sequenced $B$. endophyticus genomes are approximately 36\%, and similar to the B. endophyticus Hbe603 and other $B$. endophyticus strains used in pan-genome analysis. The complete genome of Hbe603 is $5.31 \mathrm{Mb}$ and consists of a chromosome and 8 plasmids [16]. Annotation using RAST [17], predicted the number of coding sequences of B. endophyticus Hbe603 to be 5455 that is slight higher than the sequenced genomes in this study except for 3618_1C. High numbers of accessory genes of $B$. endophyticus 3618_1C are represented in the unknown function or as hypothetical proteins. A total of 5310,
5431,5358 , and 5408 predicted coding sequences in strains 3631_9D, 3618_1C, 3631_10C and 3617_2C respectively (Table 3 ). RAST analyses showed $B$. megaterium DSM 319 to be the closest neighbor to the B. endophyticus strains with the comparative analysis using sequence similarity option.

\section{Plasmids of $B$. endophyticus}

B. endophyticus Hbe603 complete genomes consist of 8 plasmids. The roles of the plasmids have never been reported in B. endophyticus Hbe603 strain. The draft genomes of $B$. endophyticus strains sequenced in this study each presented 4-7 plasmids (Table 3, Additional file 4: Table S1). Comparative analysis of the sequenced $B$. endophyticus strains with $B$. endophyticus Hbe603 consisted of partial regions of plasmids, while pBEH1, pBEH6, and pBEH7 are the common plasmids shared. Plasmids sizes of draft genome $B$. endophyticus strains were significant smaller than the $B$. endophyticus Hbe603 plasmids (Additional file 4: Table S1). None of the $B$. endophyticus plasmids were similar with the $B$. anthracis $\mathrm{pXO} 1$ and $\mathrm{pXO} 2$ plasmids. 


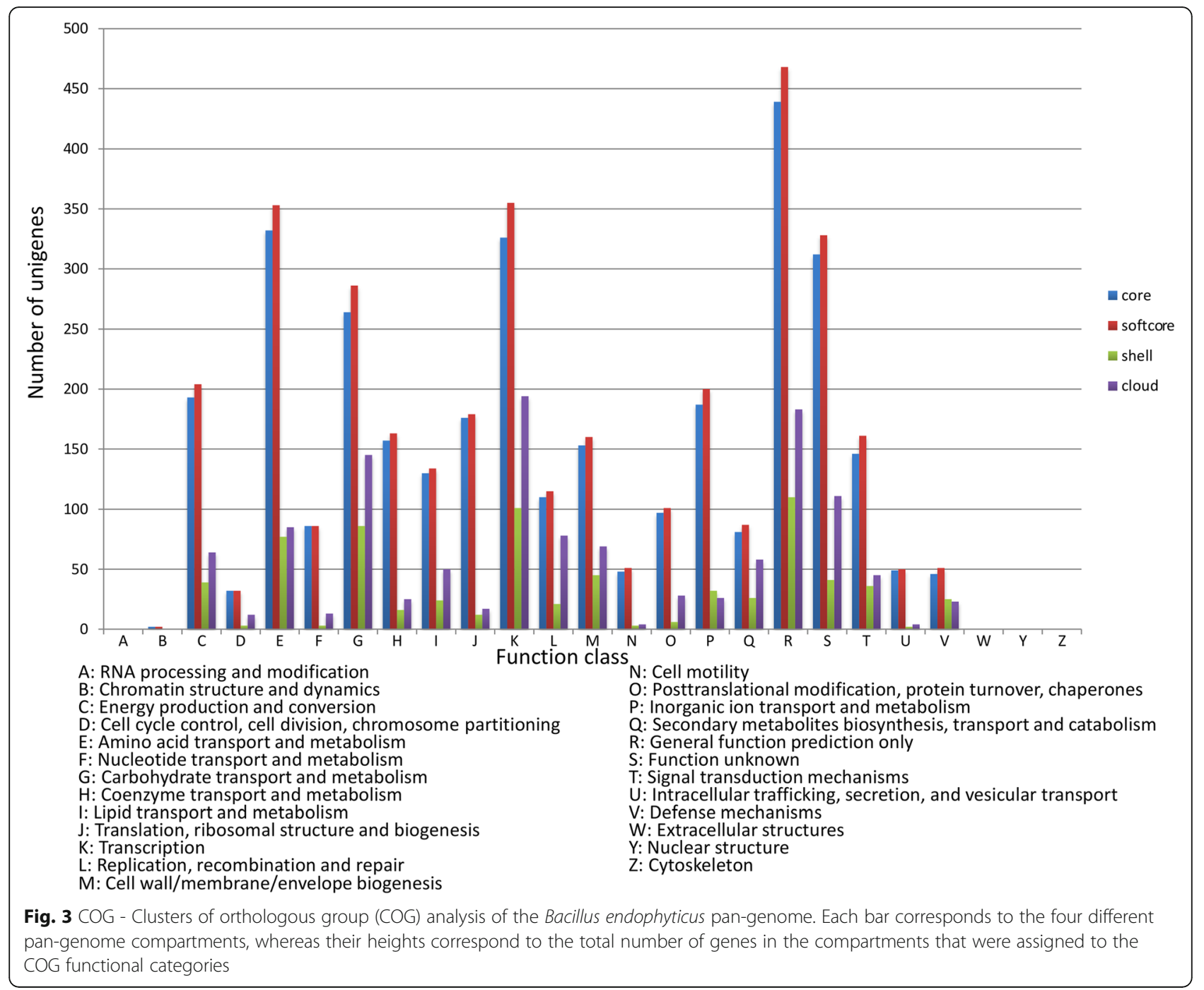

\section{Virulence, resistance and defense genes}

Coding sequences linked to antibiotic- and toxic compound resistances were identified in the $B$. endophyticus strains. Comparative analysis of $B$. endophyticus 3618_1C, 3631_9D, 3631_10C, KCTC 13922, and DSM 13796 showed unique coding sequences that include arsenical-resistance protein Acr3, copper resistance protein $\mathrm{D}$ for copper homeostasis, multidrug resistance transporter Bcr/CflA family and fosfomycin resistance protein fos $\mathrm{B}$ that are absent in the B. endophyticus Hbe603 and 2102 genomes. B. endophyticus 3617 2C strain also contained these coding sequences except the Acr3 and the multidrug resistance transporter $B c r / C f l A$ family CDS. The transcriptional regulator $N f x \mathrm{~B}$ was present in B. endophyticus 3618_1C and 3617_2C strains (i.e. absent in the other compared B. endophyticus strains in this study). This transcriptional regulator is involved in the MexC-MexD-OprJ multidrug efflux system that contributes to antibiotic- or toxic compounds resistances [18]. The genome analyses of $B$. endophyticus strains confirmed the presence of CDS for the macrolide-specific efflux protein $m a c \mathrm{~A}$ and permease protein $m a c B$ for the multidrug resistance efflux pumps, except in strains 3618_1C and 2102. The MacAB-TolC macrolide efflux transport system has mostly been studied in gram-negative bacteria. The presence of macA in the system is known to stimulate the ATPase activity of $m a c B$ to bind macrolides such as erythromycin and azithromycin. Meanwhile the overproduction of macA and macB results in an increase resistance to the macrolides antibiotics [19]. $B$. endophyticus is regarded as plant-endophytic bacterium that survives high-salt concentration [1, 13]. The sigma-M predicted to response to high concentration of salt [20] was found in the 8 compared B. endophytcicus genomes in this study. Jia et al. [16] predicted other sigma factors responsible for gene regulation in $B$. endophyticus. 


\section{Bacillus endophyticus prophages}

PHAGE_Bacill_phBC6A52 was the common intact prophage in strains 3631_9D and 3631_10C. B. endophyticus 3631_10C presented additional two partial prophage regions annotated as PHAGE_Lister_B054_NC_009812 and Bacill_1_NC_009737. The latter, PHAGE_Bacill_1_NC_ 009737, was also present in B. endophyticus 3617_2C. About 7 prophage regions were identified in B. endophyticus 3618_1C strain (Table 3). This included PHAGE_Bacill_G_NC_023719, PHAGE_Burkho_phi023719, PHAGE _Synech_S_MbCM100_NC_023584, PHAGE_Entero_phi 92_NC_023693, PHAGE_Escher_vB_EcoM_UFV13_NC 031103, PHAGE_Bacill_SP_15_NC_031245 and PHAGE _Bacil_BM5_NC_029069. The 7 prophages were also identified in B. endophyticus DSM_13,796 and KCTC 13922 except for PHAGE_Entero_phi92_NC_023693 and PHAGE_Escher_vB_EcoM_UFV13_NC_031103. However, the prophage regions differ in their sizes. Only 4 prophages were determined in the $B$. endophyticus Hbe603 reference strain, whereby most were annotated as hypothetical proteins [16]. In B. endophyticus 2102, no prophage sequence regions were identified. Comparative analysis of prophages between $B$. anthracis strains 3631_1C and 20SD [14] and B. endophyticus sequenced in this study indicated that the four Lambda $B$ a prophages remain unique to $B$. anthracis.

\section{PGA biosynthesis complex}

The PGA subunits $p g s \mathrm{~B}, p g s \mathrm{C}, p g s \mathrm{~A}$ and $\gamma$-glutamyl transpeptidase $(g g t)$, and $p g s E$ genes were present in the 4 sequenced $B$. endophyticus strains (3617_2C, 3618_1C,
3631_9D, 3631_10C) and other 4 compared B. endophyticus genomes (2102, Hbe603, KCC 13922, DSM 13796) in this study. The PGA subunits of $B$. endophyticus genomes are located in the chromosome compared to the plasmid, pXO2, of B. anthracis. In B. anthracis, the PGA subunits are presented and annotated as capBCADE (Fig. 4). They are associated with the synthesis of the poly- $\gamma$-glutamate capsule formation rather than a released PGA. Due to no capsule formation observed in the B. endophyticus strains, this suggests that PGA biosynthesis is associated in a released form. Bacillus species genomes i.e. B. subtilis, and B. licheniformis (Fig. 4) consist of pgs subunits. The amino acid sequence identities of cap/pgs subunits to B. anthracis are indicated in Fig. 4 indicating the percentages of amino acid similarities between B. endophyticus, B. anthracis and B. subtilis. B. endophyticus and $B$. subtilis synthetic pgsBCA genes are homologous to the capBCA genes of $B$. anthracis. The study identified a pgsE subunit of $B$. endophyticus, which is analog to cap $\mathrm{E}$ in B. anthracis (Fig. 4) and also referred to $y w t \mathrm{C}$ in $B$. subtilis. The subunit $p g s \mathrm{~S}(y w t \mathrm{D})$ is present in the B. subtilis and B. licheniformis PGA synthetic operon and absent from $B$. endophyticus and B. anthracis PGA synthetic operon (Fig. 4). The amino acid sequence of B. endophyticus capC is $82 \%$ similar to B. anthracis $\operatorname{cap} \mathrm{C}$, indicating a high probability of $\operatorname{cap} \mathrm{C}$ region primer annealing in either B. endophyticus or B. anthracis strains. The capsule regulons $a c p \mathrm{~A}$ and $a c p \mathrm{~B}$ in $B$. anthracis were observed on the same PGA operon. In B. endophyticus genomes, none of these two regulons were observed in the PGA complex operon (Fig. 4).

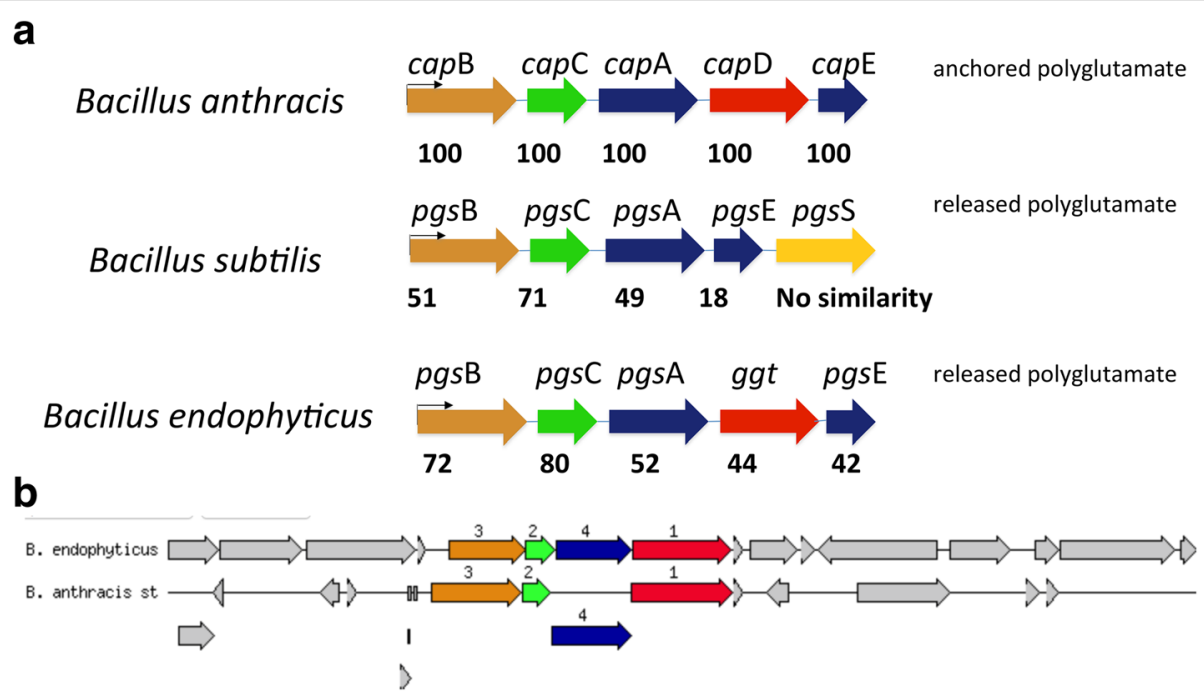

Fig. 4 Comparative structure of the polyglutamate (PGA) subunit genes of the Bacillus endophyticus 3631_9D, B. anthracis Ames and B. subtilis natto IF03336. All cap/pgs coding sequence are indicated in colours with (a) representing the comparison of the PGA synthetic operon of $B$. anthracis, B. subtilis, and B. endophyticus. Numbers indicates amino acid sequence identities (\%) of the cap/pgs proteins to those of B. anthracis. (b) Indicates the annotated sequence based comparison of the B. endophyticus 3631_9D and B. anthracis Ames PGA genes. Number 1 (red) represents pgs/capD, 2 pgs/capC, 3 (brown) pgs/capB, 4 (blue) pgs/capA 


\section{Glutamyltranspeptidases (ggt)}

An open reading frame (ORF) that encodes for $\gamma$-glutamyltranspeptidases (GGT) was present in the sequenced B. endophyticus strains (Fig. 4b) and other 4 compared B. endophyticus strains (2102, Hbe603, KCC 13922, DSM 13796). In this study, the nucleotide sequence analysis of the ggt in B. endophyticus, B. anthracis and other Bacillus species showed sequenced B. endophyticus strains cluster with the compared B. endophyticus strains (Fig. 5). Single nucleotide and amino acid variations were observed between aligned ggt of B. endophyticus and $B$. anthracis 20SD. The aligned ggt amino acid sequences of the reported B. endophyticus strains in this study are $44 \%$ identical to B. anthracis (Fig. 4). The sequenced $B$. endophyticus strains in the study had the same nucleotide identity profile with $B$. endophyticus DSM 13976 and KCTC 13922. B. endophyticus 3618_1C grouped separately amongst the other $B$. endophyticus strains, and this was also observed in the heat map (Fig. 2). There was a clear separation between the ggt of the $B$. endophyticus strains and the other Bacillus species, with the closest being B. anthracis Ames ancestor and $B$. megaterium (Fig. 4).

\section{Bacillus endophyticus and $B$. anthracis features}

The annotation of $B$. endophyticus strains and B. anthracis showed the presence of the import and iron release four-gene cluster (feuABCD) and the Fe-bacillibactin (iron carrier) uptake system common in both. The four-gene operon of $f e u \mathrm{~A}-f e u \mathrm{~B}-f e u \mathrm{C}-f e u \mathrm{D}$ and trilactone hydrolase (bacillibactin) siderophore YuiI (BesA) was identified in the $B$. endophyticus genomes. Bacillibactin siderosphore is synthesized through the alternative non-ribosomal peptide synthetase pathways and helps the bacterium in iron acquisition from their environment [21]. Genes identified in both $B$. endophyticus and $B$. anthracis also included bacitracin $\mathrm{ABC}$ transporters, bacitracin export ATP-binding protein $B c e \mathrm{~A}$ and permease protein $B c e \mathrm{~B}$, which confers resistance to bacitracin or stress response as defensive mechanisms.

\section{Discussion}

The presence of the PGA subunits $p g s /$ capA-C in the South African B. endophyticus strains isolated alongside $B$. anthracis strains from the 2009 anthrax outbreak initiated the comparative investigation of these two species. B. endophyticus and B. anthracis can be differentiated based on sensitivity to the $\gamma$-phage [13], which is not a reliable differentiating character as resistance to $\gamma$-phage had been reported amongst the normally $\gamma$-phage sensitive $B$. anthracis $[13,22]$. In this study a more comprehensive approach that included morphology, biochemical as well as WGS were used to compare these two species in order to identify differentiating characteristics for diagnostic purposes. B. endophyticus has not been reported to date to be isolated with $B$. anthracis. This and the presence of PGA genes in B. endophyticus is noteworthy since capsule genes is an important diagnostic characteristic of $B$. anthracis. B. anthracis could be differentiated from $B$. endophyticus based on unique capsulated box-shaped bacilli in long chains (in culture), $\gamma$-phage susceptibility characteristic and the presence of the toxin pag gene. B. endophyticus showed round-edged bacilli present either as single cells or in short chains, $\gamma$-phage resistant and the absences of the toxin pag gene. Identification of the $p g s / c a p$ BCA genes of the PGA biosynthetic pathways

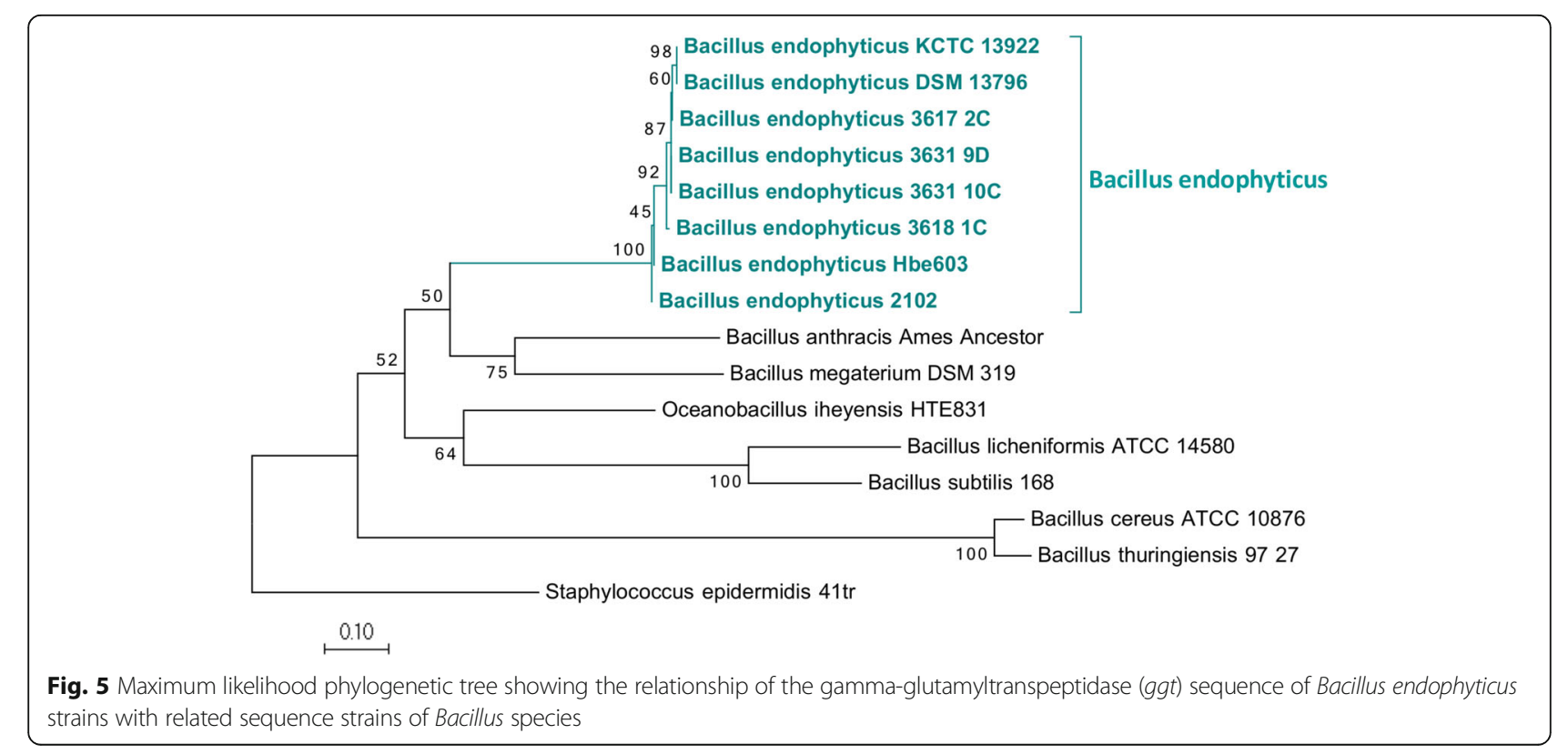


in both species using WGS comparative analysis shows the value of this approach. The pgsBCA, $\gamma$-glutamyl-transpeptidase $(g g t)$ and $p g s \mathrm{E}$ open reading frames were identified in the chromosomes of B. endophyticus genomes.

The South African B. endophyticus strains were differentiated from $B$. anthracis based on $\gamma$-phage microbiological characteristics and real-time PCR, whereas $16 \mathrm{~S}$ rRNA sequences and Omnilog identified the $B$. endophyticus strains [13]. However identification of the B. cereus sensu lato group using $16 \mathrm{~S}$ rRNA gene sequencing is often challenging, since it has been regarded as a single taxon based on similar 16Sr RNA sequences [23]. The diagnosis of $B$. anthracis require the use of microbiology characteristics as well as conventional or real-time PCR that detects $B$. anthracis specific chromosomal regions, toxin genes on pXO1 and capsule genes on pXO2 [2]. However, regions similar to the $B$. anthracis plasmids (pXO1 and pXO2) have been reported in other Bacillus species [11, 12] as observed with conventional PCR of $B$. endophyticus that amplified cap $\mathrm{A}, \operatorname{cap} \mathrm{B}$ and $\operatorname{cap} \mathrm{C}$ regions [13].

Previous studies have reported a close relationship between B. endophyticus and B. smithii [1], which was also demonstrated in this study (Table 2, Additional file 3: Figure S3). They could be differentiated based on capsule, motility and rods morphology appearance $[1,13,24]$. The WGS of B. endophyticus strains reported in the study were closely related to $B$. megaterium DSM 319 using RAST as reported in B. endophyticus 2102 WGS [25]. However, $B$. megaterium DSM 319 does not contain any plasmids unlike other B. megaterium strains [26] and this has the potential to create a bias in RAST annotations [17]. $B$. megaterium bacilli $(2.0-5.0 \mu \mathrm{m})$ are slightly larger than $B$. endophyticus $(2.5-3.5 \mu \mathrm{m})$ and both are non-motile (Table 2). Features of $B$. megaterium can be confused with $B$. anthracis as both are non-motile, encapsulated and some B. megaterium strains are non-heamolytic [27], but can be differentiated based on penicillin and $\gamma$-phage sensitivity [28]. The $\gamma$-phage sensitivity is noted in B. anthracis strains containing the $\gamma$-phage receptor GamR gene [29]. None of the sequenced and compared B. endophyticus genomes had this gene. B. endophyticus is also non-motile, non-hemolytic, and penicillin sensitive, which do not distinguish it from $B$. anthracis. B. megaterium, B. endophyticus and B. anthracis can be differentiated based on morphology followed by verification of virulence factors and/or prophage region using real-time PCR [30].

None of the lambda prophage regions of $B$. anthracis were found in $B$. endophyticus using WGS comparative analysis. As indicated the prophage regions of $B$. anthracis lambdaBa03 (01-04) accurately distinguished $B$. anthracis from $B$. endophyticus and other related Bacillus species
[30]. The B. endophyticus strains in this study presented many different prophage regions. The $B$. endophyticus strains 3618_1C shared common prophages with the $B$. endophyticus DSM_13,796 and KCTC 13922. Jia et al. [16] determined four prophage regions in the B. endophyticus Hbe603 strain, which were determined as hypothetical proteins that are different from the prophages in B. endophyticus strains reported in this study. The shared prophage regions amongst $B$. endophyticus strains can be investigated as more genomes become available that could be used in diagnostic assays.

WGS of the sequenced $B$. endophyticus strains in this study are closely related to B. endophyticus DSM 13796 and KCTC 13922 based on the average nucleotide identify (Fig. 2). The overrepresentation of the COG in the core cluster analysis might show that $B$. endophyticus has a high number of highly conserved genes and that horizontal gene transfer does not necessarily play a major role in its evolution. One key feature of $B$. endophyticus identified through WGS is the bacillibactin-associated genes for biosynthesis that are also present in $B$. anthracis and many other members of the $B$. cereus sensu lato group [21]. The bacitracin cluster of genes identified in B. endophyticus and $B$. anthracis are known to be a peptide antibiotic that is non-ribosomally synthesized in some strains of Bacillus [31], especially in B. subtilis. It has the ability to disrupt the cell wall and peptidoglycan synthesis of the gram-positive and gram-negative bacteria. However, bacillibactin and bacitracin cannot be used as differentiating features of $B$. endophyticus strains since they are also present in $B$. anthracis strains.

B. endophyticus Hbe603 consists of one chromosome and 8 plasmids that belong to the members of Bacillus group [16]. The function or the role of the plasmids has not yet been studied. Sequence comparison revealed no similarities between $B$. endophyticus and B. anthracis plasmids. The PGA complex is present in most Bacillus species including B. licheniformis [32], B. subtilis [10], $B$. anthracis [4] and B. cereus sense lato group including $B$. cereus biovar anthracis [12,33]. In this study, the PGA biosynthesis operon was also identified in the B. endophyticus genomes. The PGA subunits are located in the chromosome of the B. endophyticus strains unlike in the plasmid of B. anthracis.

The polyglutamate depolymerase cap $\mathrm{D}$ is present in the B. anthracis [7] and belongs to the $\gamma$-glutamyltransferase (GGT) family. This gene is responsible for the covalent anchoring of the capsule to peptidoglycan and act as a depolymerase in B. anthracis [7]. B. anthracis capD gene is related to $B$. subtilis natto $y w r \mathrm{D}$ and $B$. licheniformis DSM13 ggt. However, $y w r \mathrm{D}$ or ggt is located in the chromosome and reside in a locus distant from the pgsBCA subunits. The ggt and capD subunits were present in both $B$. endophyticus and B. anthracis genomes 
respectively (Fig. 4). The ggt is located on a locus adjacent to the pgs $B C A$ subunit genes in the chromosome of the sequenced $B$. endophyticus (3631_9D, 3618_1C, 3631_10C, 3617_2C) and other compared B. endophyticus genomes (2102, Hbe603, KCC 13922, DSM 13796). The ggt identified in the B. endophyticus has different nucleotide and amino acid variations with $B$. anthracis and B. subtilis. Annotation of this subunit in B. endophyticus strains showed that is not linked with the attachment of the PGA to peptidoglycan, however it's associated with the PGA biosynthesis. The identified $\gamma$-glutamyltransferase in B. endophyticus genomes may suggest that it hydrolyses PGA biosynthesis as suggested for B. subtilis ggt that hydrolyses the PGA in an exo-type manner [34]. In B. subtilis NAFM5, the GGT was shown to have hydrolysed $\gamma$-D-L PGA from the Dand L-glutamate during stationary phase through transcriptional activation [35].

The $p g s \mathrm{E}$ subunit is known to stimulate the PGA production in the presence of zinc [4]. However in B. subtilis, high concentrations of $p g s \mathrm{~B}, p g s \mathrm{C}$, and $p g s \mathrm{~A}$ were determined to form PGA in the absence of $p g s E$ [36]. There is a small ORF present in B. endophyticus (Fig. 4) strains annotated as hypothetical protein, which has the same nucleotide size (144 bp) than B. anthracis capE. Protein alignment of B. endophyticus pgs/cap E is $42 \%$ identical to capE of $B$. anthracis. This ORF may be important for the PGA biosynthesis and act as an anolog pgs/cap E since B. anthracis capE is required for PGA biosynthesis [4]. The small ORF is found after the ggt/ capD in both the B. endophyticus and B. anthracis PGA operon (Fig. 4). The B. subtilis pgsS is an exo- $\gamma$-glutamyl hydrolase that is linked to the release of PGA in the environment [4]. The $\gamma$-D-L-glutamyl hydrolase pgsS lies immediately downstream of pgsBCA genes in $B$. subtilis chromosome [37]. This subunit encode enzyme that cleaves the glutamyl bond between D- and L-glutamic acids of PGA. The $p g s$ S subunit was not identified in the B. endophyticus genomes. An ORF was identified in the PGA operon of $B$. endophyticus genomes, annotated as a putative esterase/lipase, that lies immediately downstream after the $p g s \mathrm{E}$. This putative extracellular esterase belongs to the hydrolase enzymes family that might also be involved in the hydrolysis of PGA, but this hypothesis needs further investigation. The regulatory genes, $a c p \mathrm{~A}, a c p \mathrm{~B}$ and atxA (located in pXO1) are known to control the expression of $B$. anthracis capsule PGA biosynthesis operon cap BCADE [5]. The two regulons $a c p \mathrm{~A}$ and $a c p \mathrm{~B}$ located in the pXO2 were observed in the $B$. anthracis 20SD PGA biosynthesis operon, which is absent in the $B$. endophyticus PGA operon.

The exo-polysaccharide biosynthesis ORF was identified in the $B$. endophyticus genomes. It consisted of manganese-dependent protein-tyrosine phosphatase, tyrosine-protein kinase transmembrane modulator epsC, and tyrosine-protein kinase eps $\mathrm{D}$. The tyrosine-protein kinase transmembrane modulator $E p s C$ and tyrosine-protein kinase EpsD are found in the same operon. Extracellular polysaccharides (EPS) are polymers that consist of different simple sugars. They are produced by variety of bacteria and may be assembled as capsular polysaccharides (CPS) tightly associated with the cell surface or they may be liberated into the growth medium. In E. coli and B. subtilis the epsC and eps D are reported to control UDP-glucose dehydrogenase activity [38, 39]. In B. subtilis strains, cells are held together by EPS and amyloid-like fibers for biofilm formation [40]. In B. endophyticus genomes, in the same operon of exo-polysaccharide, the UDP-glucose dehydrogenase and hyaluronan synthase enzymes were identified. Hyaluronan synthase is membrane bound enzyme that is used to produce the glycosaminoglycan hyaluronan at the cell surface through the membrane. Hyaluronan synthesis in most bacteria is associated with protecting the bacteria against host and environmental factors, which may be detrimental to survival [41]. The hyaluronic acid polysaccharide capsule was found in the Streptococcus pyrogenes [41]. In order for S. pyrogenes to synthesize a HA capsule, at least three different genes must be present and arranged in an operon designated the HA synthesis manner [41]. This includes the HA synthase and two sugar precursors (UDP-glucose dehydrogenase and UDP-glucose-pyrophosphorylase). In $B$. endophyticus genomes only one sugar precursor UDP-glucose dehydrogenase, and the hyaluronan synthase are present. The role of HA needs further investigation in the B. endophyticus strains.

\section{Conclusion}

B. endophyticus is a gram-positive, non-motile, nonhemolytic, rod-shaped bacterium which is endospore forming, penicillin sensitive but $\gamma$-phage resistant. $B$. anthracis has all these characteristics in common with $B$. endophyticus with the exception that it is $\gamma$-phage sensitive bacterium. Bacillus species, which include $B$. anthracis, $B$. megaterium, B. endophyticus and B. smithii can be differentiated based on their morphological appearances and other microbiological features. However, most of these microbiological features (biochemical tests i.e. the presence of lecitinase, starch, VP test motility and other tests) are not routinely used for identification and characterization of Bacillus species. Molecular techniques such as real-time PCR targeting species-specific chromosomal markers, virulence genes and 16S rRNA sequencing, should continuously be used to identify or distinguish related Bacillus species. This can further be supplemented with specific prophages of the bacterium or other specific genes present in the genome. B. endophyticus is considered as industrial important due to biotechnology properties like the production of antibiotics such as fosfomycin and bacitracin. 
B. endophyticus can easily be differentiated from $B$. anthracis based on the morphology appearance but confirmation of virulence factors like capsule genes identified in B. endophyticus could complicate anthrax diagnostics. Whole genome sequencing identified and differentiated $B$. anthracis and B. endophyticus PGA capsule genes. $B$. anthracis and $B$. endophyticus PGA biosynthesis subunits were determined to be located in the $\mathrm{pXO} 2$ and chromosome respectively. The B. endophyticus strains couldn't synthesize a surface associated $\gamma$-PGA, suggesting that PGA helps the bacteria to survive under adverse conditions. Therefore B. endophyticus is a non-capsulated bacterium that survives at high salt concentrations. Prophage regions have emerged as key markers in distinguishing $B$. anthracis and eliminating other related Bacillus species. The study highlights the significance of using whole genome shotgun sequencing to identify virulence and other important genes that might be present amongst unknown samples from natural outbreaks.

\section{Methods \\ Isolates}

The B. endophyticus and B. anthracis isolates included in this study were isolates collected during the 2009 anthrax outbreak in the Northern Cape Province (NCP) of South Africa. These isolates included a B. endophyticus and $B$. anthracis isolate from the same animal. The $B$. endophyticus were isolated from blood collected from animal carcasses whereas $B$. anthracis isolates were isolated from soil below the carcass as well as blood collected from animal carcasses (Table 1). The B. endophyticus isolates exhibited some similar phenotypic and genetic similarities to those of $B$. anthracis [13] and therefore we characterized these isolates to enhance and contribute to the diagnosis of $B$. anthracis. The incubation condition for $B$. endophyticus range from 10 to $55{ }^{\circ} \mathrm{C}$ although the optimum growth temperature is between 28 and $30{ }^{\circ} \mathrm{C}$, but this study used conditions specific for anthrax diagnosis as described by the International protocols for anthrax [42].

\section{Phenotypic characterization}

In this study we focused mainly on capsule characterization of $B$. endophyticus strains to enhance the phenotypic characterization previously done on South African $B$. endophyticus and B. anthracis stains [13] as well as summarizing phenotypic characterizations of related Bacillus species. Four B. endophyticus and three $B$. anthracis strains isolated from animal anthrax cases in NCP in South Africa available at Agricultural Research Council-Onderstepoort Veterinary Institute (ARC-OVI) were used in this study (Table 1 ). The B. endophyticus and $B$. anthracis isolates were collected from 2009 anthrax outbreaks in the NCP of South Africa (Table 1). The samples were processed at the ARC-OVI reference laboratory (Onderstepoort, South Africa), where B. anthracis suspected cases are confirmed. Pure cultures were grown on $5 \%$ SBTA, followed by incubation at $37^{\circ} \mathrm{C}$ for $24 \mathrm{~h}$ for the observation of colony morphology and to determine hemolytic activity [42]. Colony morphology was observed on nutrient agar containing $0.8 \%$ sodium bicarbonate following incubation in the presence of $5 \% \mathrm{CO}_{2}$ at $37^{\circ} \mathrm{C}$ for $24-48 \mathrm{~h}$ in the dark to induce capsule formation. The capsules from strains incubated on $0.8 \%$ sodium bicarbonate supplemented nutrient agar were stained using India ink, Giemsa and copper sulphate followed by visualization using light microscopy [42, 43]. Each culture was also transferred to blood serum and incubated under both aerobic and anaerobic conditions at $37{ }^{\circ} \mathrm{C}$ for $24 \mathrm{~h}$ to determine the formation of a capsule [42]. Blood smears were stained using Rapi-Diff and visualized by light microscopy. The positive control for the capsule production included B. anthracis 3618_2D (cap ${ }^{+}$, virulent strain) [13] whereas the negative controls included $B$. licherniformis ATCC 12759 (cap $^{-}$) and B. anthracis Sterne (cap ${ }^{-}$) strains. Phenotypic properties of $B$. endophyticus and $B$. anthracis were compared with those from published literature including B. megaterium and B. cereus as shown in Table 2 ([1, 24, 27, 42, 44], http://www.tgw1916.net).

\section{Genomic DNA extraction}

B. endophyticus and B. anthracis strains (Table 1) were inoculated in $2 \mathrm{ml}$ nutrient broth, followed by overnight incubation at $37^{\circ} \mathrm{C}$. The cells were harvested by centrifugation at $5000 \mathrm{xg}$ for $10 \mathrm{~min}$. Genomic DNA was extracted from the harvested cells using the DNAeasy Tissue kit (Qiagen, Germany) according to the manufacturer's instructions. The isolated DNA was then quantified using the Qubit fluorometric method (Life Technologies, USA) according to the manufacturer's instructions. The DNA integrity was monitored through electrophoreses using a $0.8 \%$ agarose gel pre-stained with ethidium bromide and visualized on a UV transilluminator.

\section{High-throughput sequencing}

Shotgun library preparation of four B. endophyticus (Table 1) strains was performed using the Nextera DNA Sample Preparation kit (Illumina, USA). Clusters generation and the sequencing were performed using the TruSeq ${ }^{\text {Twx }} \mathrm{PE}$ Cluster kit v2-cBot-HS and TruSeq SBS v3-HS (200 cycle) kit respectively (Ilumina, USA). The sequencing was performed on the HiScan SQ sequencer (Illumina, USA).

\section{Genome assembly and annotation}

Sequence data quality was assessed using FastQC software v 0:10.1 [45]. Ambiguous nucleotide sequence and sequence adapters were trimmed using CLC Genomic Workbench 7.5 (Denmark). The de novo assemblies 
were performed using the CLC Genomic Workbench 7.5. The B. endophyticus strains contigs were further extracted and analyzed with BLASTn [46] using B. endophyticus Hbe603 (Genbank accession no: CP011974) as reference genome. MAUVE tool [47] was used to order the sequence of $B$. endophyticus reported in the study using B. endophyticus Hbe603 as a reference. The assembled contigs were annotated using NCBI prokaryotic genome automatic annotation pipeline (PGAAP) and rapid annotation using subsystem technology [48] annotation server for subsystems and functional annotation [17]. The presence of prophage sequence regions in the 8 B. endophyticus genomes (3631_9D, 3631_10C, 3618_1C, 3617_2C, Hbe603, 2102, KCC 13922, and DSM 13796) were determined using PHAge Search Tool (PHAST) [49].

\section{S rRNA gene phylogenetic analysis}

The 16S rRNA sequence region consisting of approximately 1500 bases was extracted from the assembled genomes of B. endophyticus strains (3631_9D, 3618_1C, 3631_10C and 3617_2C). These sequences were further aligned and compared with the $16 \mathrm{~S}$ rRNA gene sequences of Bacillus species available in NCBI (http:www.ncbi.nlm.nih.gov). NCBI BLAST homology searches of the $16 \mathrm{~S}$ rRNA gene sequences were performed to assess homologous hits to sequences available in NCBI. Multiple alignments of the gene sequences extracted from assembled genomes and from those mined from NCBI were performed using MAFFT [50]. Maximum likelihood analysis of the B. endophyticus 16S rRNA nucleotide sequences and related Bacillus group sequences were performed using 1000 bootstrap iterations in MEGA 6.0.

\section{Average nucleotide identities, pan-genome analyses and functional classification of orthologous genes}

The CDSs (coding domain sequences) of $B$. endophyticus sequenced strains were subsequently compared against each using pair-wise BLASTn, to allow for calculations of average nucleotide identities. The pan-genome homology of all the 8 B. endophyticus (3631_9D, 3631_10C, 3618_1C, 3617_2C, Hbe603, 2102, KCC 13922, and DSM 13796) were computed using the get homologues tool [51] with default parameters. In brief, the tool conducted similarity searches between the CDSs of all 8 genomes using pair-wise BLASTp [46], and these were subsequently clustered into the different pan-genomic categories using OrthoMCL [52]. The analysis resulted in four clusters, and these were defined as: core-genes present in all genomes; softcore-genes present in $95 \%$ of the genomes; shell-genes present in few but not all genomes; and the cloud - genes present in two or less of the genomes. The core and the softcore represent sets of conserved or house keeping genes. The softcore clusters were included in the analysis because the sequenced draft genomes of $B$. endophyticus strains in this study might be missing some of the essential genes. Both the shell and the cloud compose of accessory genes that play a role towards the lifestyle and adaptation characteristics of an organism to its particular environment.

The four clusters determined for the 8 genomes were searched for shared pattern similarities against a conserved domain database of cluster of orthologous groups using rps-blast with $-\mathrm{E}<1 \mathrm{e}-3$. Genes with shared pattern similarities were assigned classes that were later categorized into the COG (clusters of orthologous group) subgroups to determine their distributions for all the clusters.

\section{Polyglutamate (PGA) subunit genes analysis}

The presences of the PGA synthesis genes were determined for the $8 \mathrm{~B}$. endophyticus strains (3631_9D, 3631_10C, 3618_1C, 3617_2C, Hbe603, 2102, KCC 13922, and DSM 13796) using analyses on the RAST server with the annotated draft genomes [17]. The PGA-capsule subunits were extracted from the annotated contigs of the $B$. endophyticus genomes using comparative analysis of RAST. B. anthracis PGA capsule subunits were compared with the B. endophyticus PGA subunits using the same annotation system. BLASTp [46] was used to compare the PGA proteins of B. anthracis, B. endophyticus and B. subtilis. Phylogenetic tree analysis of the subunit gene $\operatorname{cap} \mathrm{D} /$ $p g s \mathrm{D}$ of $B$. endophyticus, $B$. anthracis and other closely related species was constructed using maximum-likelihood. Multiple alignments of the gene sequences were constructed using multiple sequence alignment based on fast fourier (MAFFT) [50]. Alignment of the corresponding amino acid sequences was performed using CLC Genomic Workbench 7.5. MEGA 6.0 was used to construct the phylogenetic tree using 1000 bootstrap iterations.

\section{Genome sequences and accession numbers}

The four sequenced genomes of B. endophyticus were deposited in the Genbank genome database under accession numbers: B. endophyticus 3631_9D LVYL00000000, B. endophyticus 3631_10C LVYK00000000, B. endophyticus 3618_1C LWAI00000000 and B. endophyticus 3617_2C LWAG00000000. The additional four genomes that were used in the comparative analysis of $B$. endophyticus strains were retrieved from NCBI genebank. Accession numbers: B. endophyticus Hbe603 GCA_000972245.3, B. endophyticus 2102 GCA_000283255.1, B. endophyticus DSM 13,796 GCA_900115845.1 and B. endophyticus KCTC 13922 GCA_001590825.1. The sequenced B. endophyticus genome sequences in the study were further compared to the South African B. anthracis 20SD and 3631_1C strains (Genbank accession $\mathrm{nr}$ LGCC00000000 and LGCD00000000). 


\section{Additional files}

Additional file 1: Figure S1. (1) Colony morphology of (a) Bacillus endophyticus that is small circular, wet and non-mucoid and (b) $B$. anthracis appear circular, mucoid on nutrient agar supplemented with sodium bicarbonate at $5 \% \mathrm{CO}_{2}$ after incubation at $37^{\circ} \mathrm{C}$. Colony morphology of B. endophyticus and B. anthracis on sheep blood agar incubated at $37^{\circ} \mathrm{C}$. B. anthracis shows the characteristic shiny, rough with ground-glass appearance compared to the white slimy and smooth colonies of $B$. endophyticus. (TIFF $2652 \mathrm{~kb}$ )

Additional file 2: Figure S2. Phenotypic electron microscopic examination of the morphology of B. endophyticus strains after $24 \mathrm{~h}$ incubation on nutrient agar containing $0.8 \%$ sodium bicarbonate stained using copper sulphate. (TIFF $4206 \mathrm{~kb}$ )

Additional file 3: Figure S3. Phylogenetic tree of $16 \mathrm{~S}$ ribosomal RNA sequence of the Bacillus endophyticus 3618_1C, 3631_9D, 3617_2C and 3631 10C strains with related Bacillus species using maximum likelihood. Geobacillus thermoglucosidasius was used as an out-group. Bootstrap values $>60$ are indicated at the internodes. (TIFF $219 \mathrm{~kb}$ )

Additional file 4: Table S1. Plasmid comparison of the four sequenced Bacillus endophyticus strains (3618_1C, 3631_9D, 3631_10C, 3617_2C) with B. endophyticus Hbe603 strain. (DOC 18 kb)

\section{Abbreviations}

ANI: Average nucleotide identity; ARC-OVI: Agricultural Research CouncilOnderstepoort Veterinary Institute; BLAST: Basic local alignment search tools; BLASTn: Basic local alignment search tool nucloetide; BLASTp: Basic local alignment search tool protein; CAP: Capsule; CDSs: Coding domain sequences; COG: Clusters of orthologous group; CPS: Capsular polysaccharides; EPS: Extracellular polysaccharides; GGTgamma: Glutamyltranspeptidases; MAFFT: Multiple sequence alignment based on fast fourier; MAUVE: Multiple alignment of conserved genomic sequence with rearrangements; NA: Not available; $\mathrm{NaCl}$ : Sodium chloride; NCBI: National centre for biotechnology information; NCP: Northern Cape province; NGS: Next generation sequencing; ORF: Open reading frame; PCR: Polymerase chain reaction; PGA: Polyglutamte; PGAAP: Prokaryotic genome automatic annotation pipeline; PGS: Polyglutamate synthase; RAST: Rapid annotations using subsystems technology; WGS: Whole genome sequencing; $\gamma$ : Gamma

\section{Acknowledgements}

We are grateful to the National Research Foundation (NRF), NRF-THRIP, Agricultural Research Council-Biotechnology Platform and AgriSETA for financial support.

\section{Funding}

This study was funded by the National Research Foundation (NRF), NRFTHRIP, Agricultural Research Council-Biotechnology Platform and AgriSETA for financial support. NRF, NRF-THRIP and AgriSETA assisted with running of the experiments, while ARC-Biotechnology provided funding for sequencing and bioinformatics support. The funding organizations only supplied funds and did not review or supply any other input.

\section{Availability of data and materials}

All data generated or analysed during this study are included in this published article [and its supplementary information files].

\section{Endnotes}

Not applicable.

\section{Authors' contributions}

KEL participated for running the experiments and writing the manuscript. OKIB assisted in running some of the bioinformatics analysis. JM and JR assisted in design of the project and drafting the manuscript. EM, FC and $\mathrm{HvH}$ participated in the design of the study, drafting the manuscript, revising it critically and provided funding. All authors have read and approved the manuscript.
Ethics approval and consent to participate

No animals involved, not applicable.

\section{Consent for publication}

Not applicable.

\section{Competing interests}

The authors declare that they have no competing interests.

\section{Publisher's Note}

Springer Nature remains neutral with regard to jurisdictional claims in published maps and institutional affiliations.

\section{Author details}

1The Biotechnology Platform, Agricultural Research Council, Private Bag X5, Onderstepoort 0110, South Africa. ${ }^{2}$ Department of Veterinary Tropical Diseases, University of Pretoria, Private bag X4, Onderstepoort 0110, South Africa. ${ }^{3}$ Bacteriology section, Agricultural Research Council-Onderstepoort Veterinary Institute, Private Bag X5, Onderstepoort 0110, South Africa.

${ }^{4}$ College of Agriculture and Environmental Sciences, University of South Africa, Florida Campus, Christiaan De Wet/ Pioneer Dr, P.O. Box X6, Florida 1710, South Africa. ${ }^{5}$ Department of Biochemistry and Microbiology, University of Zululand, Private Bag X1001, KwaDlangezwa 3886, South Africa.

Received: 30 November 2017 Accepted: 20 June 2018

Published online: 09 July 2018

\section{References}

1. Reva ON, Smirnov W, Pettersson B, Priest FG. Bacillus endophyticus sp. nov. isolated from the inner tissues of cotton plants (Gossypium sp.). Int J Syst Evol Microbiol. 2002;52(Pt 1):101-7.

2. Health WOfA. Manual of diagnostic tests and vaccines for terrestrial animals: mammals, birds and bees. In: Biological Standards Commission, World Organization for Animal Health Paris, France; 2012

3. Makino S, Uchida I, Terakado N, Sasakawa C, Yoshikawa M. Molecular characterization and protein analysis of the cap region, which is essential for encapsulation in Bacillus anthracis. J Bacteriol. 1989;171(2):722-30.

4. Candela T, Fouet A. Poly-gamma-glutamate in bacteria. Mol Microbiol. 2006; 60(5):1091-8.

5. Drysdale M, Bourgogne A, Hilsenbeck SG, Koehler TM. atXA controls Bacillus anthracis capsule synthesis via acpA and a newly discovered regulator, acpB. J Bacteriol. 2004;186(2):307-15.

6. Drysdale M, Heninger S, Hutt J, Chen Y, Lyons CR, Koehler TM. Capsule synthesis by Bacillus anthracis is required for dissemination in murine inhalation anthrax. EMBO J. 2005:24(1):221-7.

7. Candela T, Fouet A. Bacillus anthracis CapD, belonging to the gammaglutamyltranspeptidase family, is required for the covalent anchoring of capsule to peptidoglycan. Mol Microbiol. 2005;57(3):717-26.

8. Mesnage S, Tosi-Couture E, Mock M, Gounon P, Fouet A. Molecular characterization of the Bacillus anthracis main S-layer component: evidence that it is the major cell-associated antigen. Mol Microbiol. 1997:23(6):1147-55.

9. Candela T, Mock M, Fouet A. CapE, a 47-amino-acid peptide, is necessary for Bacillus anthracis polyglutamate capsule synthesis. J Bacteriol. 2005;187(22): 7765-72

10. Ashiuchi M, Soda K, Misono H. A poly-gamma-glutamate synthetic system of Bacillus subtilis IFO 3336: gene cloning and biochemical analysis of polygamma-glutamate produced by Escherichia coli clone cells. Biochem Biophys Res Commun. 1999;263(1):6-12.

11. Rasko DA, Rosovitz MJ, Okstad OA, Fouts DE, Jiang L, Cer RZ, Kolsto AB, Gill SR, Ravel J. Complete sequence analysis of novel plasmids from emetic and periodontal Bacillus cereus isolates reveals a common evolutionary history among the B. Cereus-group plasmids, including Bacillus anthracis pXO1. J Bacteriol. 2007;189(1):52-64.

12. Klee SR, Brzuszkiewicz EB, Nattermann H, Bruggemann H, Dupke S, Wollherr A, Franz T, Pauli G, Appel B, Liebl W, et al. The genome of a Bacillus isolate causing anthrax in chimpanzees combines chromosomal properties of $\mathrm{B}$. Cereus with B. Anthracis virulence plasmids. PLoS One. 2010;5(7):e10986.

13. Lekota KE, Hassim A Mafofo J, Rees J, Muchadeyi FC, Van Heerden $H_{\text {, }}$ Madoroba E. Polyphasic characterization of Bacillus species from anthrax 
outbreaks in animals from South Africa and Lesotho. J Infect Dev Ctries. 2016;10(8):814-23.

14. Lekota KE, Mafofo J, Madoroba E, Rees J, van Heerden H, Muchadeyi FC. Draft genome sequences of two south African Bacillus anthracis strains. Genome Announc. 2015;3:6.

15. Makarova KS, Wolf YI, Koonin EV. Comparative genomics of defense systems in archaea and bacteria. Nucleic Acids Res. 2013;41(8):4360-77.

16. Jia N, Du J, Ding MZ, Gao F, Yuan YJ. Genome sequence of Bacillus endophyticus and analysis of its companion mechanism in the Ketogulonigenium vulgareBacillus strain consortium. PLoS One. 2015;10(8):e0135104.

17. Aziz RK, Bartels D, Best AA, DeJongh M, Disz T, Edwards RA, Formsma K, Gerdes S, Glass EM, Kubal M, et al. The RAST server: rapid annotations using subsystems technology. BMC Genomics. 2008;9:75.

18. Gotoh N, Tsujimoto H, Tsuda M, Okamoto K, Nomura A, Wada T, Nakahashi M, Nishino T. Characterization of the MexC-MexD-OprJ multidrug efflux system in DeltamexA-mexB-oprM mutants of Pseudomonas aeruginosa. Antimicrob Agents Chemother. 1998;42(8):1938-43.

19. Xu Y, Sim SH, Song S, Piao S, Kim HM, Jin XL, Lee K, Ha NC. The tip region of the MacA alpha-hairpin is important for the binding to TolC to the Escherichia coli MacAB-TolC pump. Biochem Biophys Res Commun. 2010; 394(4):962-5.

20. Horsburgh MJ, Moir A. Sigma M, an ECF RNA polymerase sigma factor of Bacillus subtilis 168 , is essential for growth and survival in high concentrations of salt. Mol Microbiol. 1999;32(1):41-50.

21. Hotta K, Kim CY, Fox DT, Koppisch AT. Siderophore-mediated iron acquisition in Bacillus anthracis and related strains. Microbiology. 2010; 156(Pt 7):1918-25.

22. Odendaal MW, Pieterson PM, de Vos V, Botha AD. The biochemical, morphological and virulence profiles of Bacillus anthracis isolated in the Kruger National Park. Onderstepoort J Vet Res. 1991;58(1):21-6.

23. Helgason E, Okstad OA, Caugant DA, Johansen HA, Fouet A, Mock M, Hegna I, Kolsto AB. Bacillus anthracis, Bacillus cereus, and Bacillus thuringiensis-one species on the basis of genetic evidence. Appl Environ Microbiol. 2000;66(6):2627-30

24. Sumathi C, Jayashree S, Sekaran G: Screening and evaluation of antimicrobial activity of tannery fleshing utilizing fish gut bacteria. 2012.

25. Lee YJ, Lee SJ, Kim SH, Lee SJ, Kim BC, Lee HS, Jeong H, Lee DW. Draft genome sequence of Bacillus endophyticus 2102. J Bacteriol. 2012;194(20):5705-6.

26. Eppinger M, Bunk B, Johns MA, Edirisinghe JN, Kutumbaka KK, Koenig SS, Creasy HH, Rosovitz MJ, Riley DR, Daugherty S, et al. Genome sequences of the biotechnologically important Bacillus megaterium strains QM B1551 and DSM319. J Bacteriol. 2011;193(16):4199-213.

27. Beesley CA, Vanner CL, Helsel LO, Gee JE, Hoffmaster AR. Identification and characterization of clinical Bacillus spp. isolates phenotypically similar to Bacillus anthracis. FEMS Microbiol Lett. 2010;313(1):47-53.

28. Turnbull, PC. Definitive identification of Bacillus anthracis-a review. J Appl Microbiol. 1999;87(2);237-40

29. Davison S, Couture-Tosi E, Candela T, Mock M, Fouet A. Identification of the Bacillus anthracis (gamma) phage receptor. J Bacteriol. 2005:187(19):6742-9.

30. Agren J, Hamidjaja RA, Hansen T, Ruuls R, Thierry S, Vigre H, Janse I, Sundstrom A, Segerman B, Koene M, et al. In silico and in vitro evaluation of PCR-based assays for the detection of Bacillus anthracis chromosomal signature sequences. Virulence. 2013:4(8):671-85.

31. Johnson BA, Anker H, Meleney FL. Bacitracin: a new antibiotic produced by a member of the B. Subtilis group. Science. 1945;102(2650):376-7.

32. McLean RJ, Beauchemin D, Clapham L, Beveridge TJ. Metal-binding characteristics of the gamma-Glutamyl capsular polymer of Bacillus licheniformis ATCC 9945. Appl Environ Microbiol. 1990;56(12):3671-7.

33. Hoffmaster AR, Ravel J, Rasko DA, Chapman GD, Chute MD, Marston CK, De $B K$, Sacchi CT, Fitzgerald C, Mayer LW, et al. Identification of anthrax toxin genes in a Bacillus cereus associated with an illness resembling inhalation anthrax. Proc Natl Acad Sci U S A. 2004;101(22):8449-54.

34. Uchida I, Makino S, Sasakawa C, Yoshikawa M, Sugimoto C, Terakado N. Identification of a novel gene, dep, associated with depolymerization of the capsular polymer in Bacillus anthracis. Mol Microbiol. 1993;9(3):487-96.

35. Kimura K, Tran LS, Uchida I, Itoh Y. Characterization of Bacillus subtilis gamma-glutamyltransferase and its involvement in the degradation of capsule poly-gamma-glutamate. Microbiology. 2004;150(Pt 12):4115-23.

36. Yamashiro D, Yoshioka M, Ashiuchi M. Bacillus subtilis pgsE (formerly ywtC) stimulates poly-gamma-glutamate production in the presence of zinc. Biotechnol Bioeng. 2011;108(1):226-30.
37. Ashiuchi M, Misono H. Biochemistry and molecular genetics of polygamma-glutamate synthesis. Appl Microbiol Biotechnol. 2002;59(1):9-14.

38. Grangeasse C, Obadia B, Mijakovic I, Deutscher J, Cozzone AJ, Doublet P. Autophosphorylation of the Escherichia coli protein kinase Wzc regulates tyrosine phosphorylation of Ugd, a UDP-glucose dehydrogenase. J Biol Chem. 2003;278(41):39323-9.

39. Elsholz AK, Wacker SA, Losick R. Self-regulation of exopolysaccharide production in Bacillus subtilis by a tyrosine kinase. Genes Dev. 2014; 28(15):1710-20.

40. Branda SS, Gonzalez-Pastor JE, Dervyn E, Ehrlich SD, Losick R, Kolter R. Genes involved in formation of structured multicellular communities by Bacillus subtilis. J Bacteriol. 2004;186(12):3970-9.

41. Weigel PH. Functional characteristics and catalytic mechanisms of the bacterial hyaluronan synthases. IUBMB Life. 2002;54(4):201-11.

42. Organization WH, Epizootics IOo: Anthrax in humans and animals: World Health Organization; 2008.

43. Markey BK, Leonard FC, Archambault M, Cullinane A, Maguire D. Clinical Veterinary. In: Microbiology, 2nd edition. Edinburgh: Elsevier; 2013. p. 901.

44. Buck CA, Anacker RL, Newman FS, Eisenstark A. Phage isolated from lysogenic Bacillus anthracis. J Bacteriol. 1963;85:1423-30.

45. Andrews S: FastQC: a quality control tool for high throughput sequence data. 2010.

46. Altschul SF, Gish W, Miller W, Myers EW, Lipman DJ. Basic local alignment search tool. J Mol Biol. 1990;215(3):403-10.

47. Darling AC, Mau B, Blattner FR, Perna NT. Mauve: multiple alignment of conserved genomic sequence with rearrangements. Genome Res. 2004; 14(7):1394-403.

48. Gierczynski R, Kaluzewski S, Rakin A, Jagielski M, Zasada A, Jakubczak A, Borkowska-Opacka B, Rastawicki W. Intriguing diversity of Bacillus anthracis in eastern Poland-the molecular echoes of the past outbreaks. FEMS Microbiol Lett. 2004;239(2):235-40.

49. Zhou Y, Liang Y, Lynch KH, Dennis JJ, Wishart DS. PHAST: a fast phage search tool. Nucleic Acids Res. 2011:39(Web Server issue):W347-52.

50. Katoh K, Standley DM. MAFFT multiple sequence alignment software version 7: improvements in performance and usability. Mol Biol Evol. 2013; 30(4):772-80.

51. Contreras-Moreira B, Vinuesa P. GET_HOMOLOGUES, a versatile software package for scalable and robust microbial pangenome analysis. Appl Environ Microbiol. 2013;79(24):7696-701.

52. Fischer S, Brunk BP, Chen F, Gao X, Harb OS, lodice JB, Shanmugam D, Roos DS, Stoeckert CJ Jr. Using OrthoMCL to assign proteins to OrthoMCL-DB groups or to cluster proteomes into new ortholog groups. Curr Protoc Bioinformatics. 2011;Chapter 6(Unit 6 12):11-9.

Ready to submit your research? Choose BMC and benefit from

- fast, convenient online submission

- thorough peer review by experienced researchers in your field

- rapid publication on acceptance

- support for research data, including large and complex data types

- gold Open Access which fosters wider collaboration and increased citations

- maximum visibility for your research: over $100 \mathrm{M}$ website views per year

At BMC, research is always in progress.

Learn more biomedcentral.com/submissions 